\title{
150. Generator Potential of the Infrared Receptor of Crotaline Snakes
}

\author{
By Shin-ichi Terashima, Richard C. Goris, and Yasuji Katsuki \\ Department of Physiology, Tokyo Medical and Dental University, Tokyo
}

(Comm. by Yas Kuno, M.J.A., Sept. 12, 1967)

The physiological properties of the loreal pits of Crotaline snakes have been investigated from a behavioral point of view by Noble and Schmidt, ${ }^{1)}$ and by electro-physiological recording by Bullock and Diecke ${ }^{2)}$ and Goris and Nomoto. ${ }^{3)}$ The organs were proven beyond a doubt to respond to infrared radiation, the sensory part being the membrane at the bottom of the external cavity. Bleichmar and De Robertis ${ }^{4}$ illustrated with electron micrographs the high concentration of unmyelinated nerve endings in this membrane. However, to date no work has been reported on the generator mechanism of the impulses which are recorded from the nerves leading to this organ. In the present work we have succeeded in recording a slow potential change from the membrane in response to infrared stimulation.

The cornified epidermis was stripped from the external surface of the pit membrane of 61 curarized habu vipers, Trimeresurus $f$. favoviridis, and glass capillary electrodes $(3 \mathrm{M} \mathrm{KCl})$ with a tip diameter of $0.5 \mu$ or less and a resistance of $30-40 \mathrm{M} \Omega$ were inserted at roughly $60^{\circ}$ to the exposed nerve layer. The ground electrode was attached to the exposed muscles anterior to the heart. A grid current of roughly $5 \times 10^{-12} \mathrm{~mA}$ flowed in the circuit. The nerves were stimulated by an incandescent microscope light placed at a distance of $20 \mathrm{~cm}$ from the pit. Intensity of stimulus was varied by interposing a series of neutral filters $(50,25,10,5$, and $1 \%$ transmission) between the light source and the membrane. The transmission spectrum of these filters was essentially flat down to $2.7 \mu$, after which it dropped sharply. At this distance of $20 \mathrm{~cm}$, the lamp produced at the pit an energy of $9.74 \mathrm{~mW} / \mathrm{cm}^{2} / \mathrm{sec}$. The human hand at a room temperature of $20^{\circ} \mathrm{C}$ and a distance of $10 \mathrm{~cm}$ produces $4.77 \mathrm{~mW} / \mathrm{cm}^{2} / \mathrm{sec}$, which is nearly the same as the lamp with a $50 \%$ filter. Duration of stimulus was controlled by a Wollensak camera shutter and recorded through the use of a simple phototransistor. Although clear potential changes were observed and photographed on the oscilloscope, in general a series of 100 stimuli and responses were recorded on magnetic tape and averaged out on a TMC Computor 
of Average Transients (CAT 400 B) in order to eliminate the effects of instrument potential drift and background noise. Spikes recorded superimposed on the slow potential were also counted in this manner.

Three patterns of response were recorded: i.e., slow potential change only (Fig. 2), spikes (usually single unit) only, and spikes superimposed on a slow potential change (Fig. 1). The slow potential
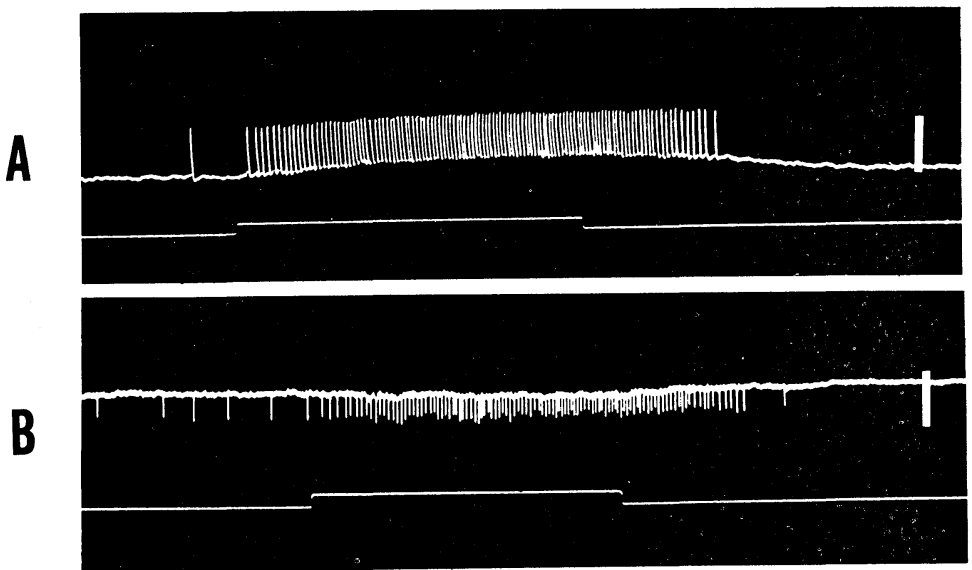

Fig. 1. Potential changes recorded from the infrared receptor membrane of Trimeresurus $f$. flavoviridis under stimulation. A: positive slow potential with superimposed spikes. Vertical bar at right of signal trace indicates $3 \mathrm{mV}$. Lower trace indicates stimulus (duration, $1 \mathrm{sec}$ ). $\mathrm{B}$ : the same as $\mathrm{A}$, but negative.

change was usually in a negative direction and of a magnitude of about $500 \mu \mathrm{V}$. The peak value of this change varied in close proportion to the intensity of the stimulus. In other words, a neutral filter of $50 \%$ reduced the change to approximately half that of an

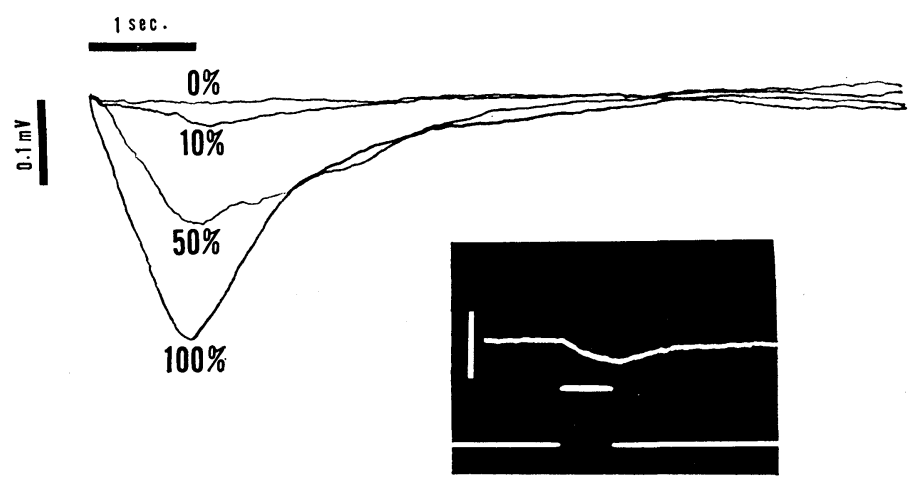

Fig. 2. Reduction of the slow potential change in response to successively reduced stimuli. Percentages indicate \% transmittance of the filters used. Each trace represents the average of 100 traces obtained from a CAT computor. Inset shows a typical slow potential without spikes. Vertical calibration, $1 \mathrm{mV}$; duration of stimulus, 1 sec. 
unfiltered stimulus (Fig. 2).

After a potential was recorded, progressively stronger concentrations of tetrodotoxin $\left(1 \times 10^{-6}, 1 \times 10^{-5}, 1 \times 10^{-4}\right)$ and procaine $\left(1 \times 10^{-3}\right.$, $1 \times 10^{-2}$ ) in Ringer's solution were injected into the cavity in such a way as to completely bathe the membrane, and then sponged out after about 3 minutes. Recordings were taken at 5 min intervals for 1 hour. The membrane was then washed with Ringer's solution, a stronger solution was injected, and another series of recordings was made, etc. A control series of recordings was made with Ringer's solution alone.

After the use of these chemicals, spike potentials disappeared completely. This is of no significance, for the same phenomenon was observed in the control recordings with Ringer's alone. Moreover, even after the lapse of one hour, no change was visible in the slow potential with either tetrodotoxin or procaine. Even with the cornified layer removed, the free nerve endings are still covered by the granular layer of the epithelium and embedded in connective tissue, so that sufficient contact with the chemicals was impossible. Because of the difficulty of isolating these nerve endings, pharmacological experiments such as those performed by other workers in this field ${ }^{5), 6)}$ are impractical.

Spikes: Both positive and negative spikes of about $1 \mathrm{mV}$ were recorded, but this polarity was not necessarily constant, since during a single recording the spikes would sometimes reverse polarity. A spontaneous firing level (i.e., in the absence of special stimulation) of 4-6 impulses/sec was observed. At peak levels of stimulation, the spikes reached a peak frequency of $83 / \mathrm{sec}$ with complete silence on cessation of stimulation. As the energy level of the stimulus was reduced with neutral filters, the number of spikes in the response was gradually reduced together with the duration of the post-stimulatory silence, to the point where response became indistinguishable from the level of spontaneous firing, and the post-stimulatory silence became non-existent.

Relation of spikes to slow potential change: When spikes were recorded superimposed on the slow potential change, the potential preceeded slightly the onset of response firing. This was also true of the curves obtained by averaging out on the CAT computor the potential change of a series of response patterns. Although the onset of the slow potential change coincided with the beginning of stimulation, return to the pre-stimulus level was significantly delayed after the cessation of stimulus. With the stimuli used, spike frequency reached its maximum near the maximum of the slow 
potential change, and diminished rapidly with the decay of the potential. A short period of silence preceeded the return of the potential to the pre-stimulus level.

As a control, recordings were attempted from the pit membrane without removing the external cornified layer, as well as from the supramaxillary branches of the trigeminal nerve feeding the membrane. In no case was a similar slow potential observed. Moreover, when the experimental animal died during recording, the slow potential disappeared simultaneously with the cessation of blood circulation in the membrane.

In conclusion, we have recorded from the receptor site a slow potential intimately connected with both stimuli and action potentials. This potential also shows the other characteristics of a generator potential, namely, that it is graded in proportion to the stimulus and is non-propagated. It is safe, therefore, to conclude that this slow potential change is the generator potential of the transducer process of the snake infrared receptor mechanism.

\section{References}

1) G. K. Noble and A. Schmidt: Proc. Amer. Phil. Soc., 77, 263-388 (1937).

2) T. H. Bullock and F. P. J. Diecke: J. Physiol. (Lond.), 134, 47-87 (1956).

3) R. C. Goris and M. Nomoto: Infrared Reception in Oriental Crotaline Snakes (to appear).

4) H. Bleichmar and E. De Robertis: Z. Zellforsh., 56, 748-761 (1962).

5) K. Nishi and M. Sato: J. Physiol., 184, 376-386 (1966).

6) T. Furukawa, T. Sasaoka, and Y. Hosoya: Jap. J. Physiol., 9, 143-152 (1959). 